\title{
Risk factors of middle lobe bronchus kinking following right upper lobectomy
}

\author{
Takahiro Yanagihara^, Yasuharu Sekine, Kazuto Sugai, Tomoyuki Kawamura, Naoki Maki, Yusuke Saeki, \\ Shinsuke Kitazawa, Naohiro Kobayashi, Shinji Kikuchi, Yukinobu Goto, Hideo Ichimura, Yukio Sato \\ Department of Thoracic Surgery, Faculty of Medicine, University of Tsukuba, Tsukuba, Japan \\ Contributions: (I) Conception and design: T Yanagihara; (II) Administrative support: Y Sato; (III) Provision of study materials or patients: T \\ Yanagihara, Y Sekine, K Sugai, T Kawamura, Y Saeki, S Kitazawa, N Kobayashi, S Kikuchi, Y Goto, H Ichimura, Y Sato; (IV) Collection and \\ assembly of data: T Yanagihara; (V) Data analysis and interpretation: T Yanagihara, N Maki, N Kobayashi; (VI) Manuscript writing: All authors; (VII) \\ Final approval of manuscript: All authors. \\ Correspondence to: Yukio Sato, MD, PhD. Department of Thoracic Surgery, Faculty of Medicine, University of Tsukuba, 1-1-1 Tennodai, Tsukuba, \\ Ibaraki, Japan. Email: ysato@md.tsukuba.ac.jp.
}

Background: The incidence rate of kinking of the middle lobe bronchus following right upper lobectomy is higher compared to that with residual lung bronchus following other lobectomies. Bronchial kinking was presumed to be caused by the displacement of the residual lung lobes, but its etiology is unclear. Moreover, prevention methods and effective treatments have not yet been established. The purpose of this study was to investigate the risk factors and etiology of middle lobe bronchus kinking and discuss prevention methods.

Methods: Patients who underwent right upper lobectomy in our hospital were retrospectively evaluated. Patient clinical characteristics, lung function, and lung lobe volume, surgical procedure were analyzed in association with the incidence of middle lobe bronchus kinking. The association between the displacement of residual lung lobes after operation and the incidence of middle lobe bronchus kinking was analyzed to assess the etiology.

Results: A total of 175 patients were enrolled in the risk analysis. Middle lobe bronchus kinking was observed in 5 patients (2.9\%). The low percentage of forced expiratory volume percentage in 1 second $(\mathrm{P}=0.021)$, the low volume ratio of the right middle lobe $(\mathrm{RML})$ to the right thoracic cavity $(\mathrm{RTC})(\mathrm{P}=0.016)$, and the low volume ratio of RML to right upper lobe (RML/RUL) (P=0.006) were significant risk factors of middle lobe bronchus kinking. In the patients who underwent CT at 6 months after surgery, the degree of the cranial displacement of RML was associated with the incidence of middle lobe bronchus kinking $(\mathrm{P}=0.025)$.

Conclusions: The risk of middle lobe bronchus kinking could be assessed preoperatively by calculating the volume ratio of RML/RTC and RML/RUL. The displacement of RML could be associated with the incidence of middle lobe bronchus kinking.

Keywords: Bronchial kink; bronchial kinking; middle lobe atelectasis; lung volume ratio; lung volume

Submitted Jan 14, 2021. Accepted for publication Apr 09, 2021.

doi: $10.21037 /$ jtd-21-105

View this article at: http://dx.doi.org/10.21037/jtd-21-105

$\wedge$ ORCID: 0000-0001-5047-6558. 


\section{Introduction}

Pulmonary lobectomy is performed as a standard treatment for lung cancer (1). The major complication after pulmonary lobectomy is prolonged air leakage, pneumonia, atelectasis, and acute respiratory distress syndrome $(2,3)$. Postoperative atelectasis occurs due to mucus plugging, bronchial edema, and bronchial obstructions such as lobar torsion and bronchial kinking, which are accompanied to anatomical displacement of the affected lobe. Especially, the incidence of lobar torsion and bronchial kinking in the right middle lobe (RML) following a right upper lobectomy is more frequent compared to such incidence after other lobectomies $(4,5)$. Lobar torsion is an emergent complication leading to infarction or congestion due to the obstruction of blood flow to the affected lobe. Lobar torsion is indicated by the findings of tortuous bronchus on bronchoscopy or radiologic findings such as tortuous obstruction of pulmonary vessels with progressive consolidation, rather than a finding of simple bronchial obstruction (6).

The risk factors of postoperative lobar torsion have been reported as: (I) lobar deflation, (II) a long lobar pedicle, (III) presence of a complete fissure, (IV) pneumothorax or pleural effusion, and (V) mobilization of the inferior pulmonary ligament, which suggests that the mobility of residual lung lobe causes postoperative lobar torsion (7). On the other hand, bronchial kinking was expected to be caused by the displacement of the residual lung lobes. The distance between the carina and middle lobe orifice was reported as a risk factor of middle lobe bronchus kinking, but the etiology was unclear (8). Bronchial kinking causes not only a persistent cough, breathlessness, and inflammation but also atelectasis, pneumonia, and a decrease of pulmonary function; however, prevention methods and effective treatments have not been established $(4,8,9)$. Moreover, mere changes of bronchial angle, without obstruction or stenosis, can also induce the clinical symptoms and a reduction of postoperative pulmonary function, which suggests that the symptoms and functional loss could persist long term (9-11). Thus, investigating the risk factors and etiology of bronchial kinking is important to discuss prevention methods and treatments that could lead to improved long-term quality of life following a lobectomy.

Therefore, we investigated the risk factors of middle lobe bronchus kinking and the displacement of the residual lobes after right upper lobectomy, focusing on the volume of each lung lobe. The purpose of this study was to investigate the risk factors and etiology of middle lobe bronchus kinking following right upper lobectomy.

We present the following article in accordance with the STROBE reporting checklist (available at http://dx.doi. org/10.21037/jtd-21-105).

\section{Methods}

\section{Patients}

We retrospectively evaluated the patients who underwent right upper lobectomy at our hospital from January 2011 to December 2017. Preoperatively, all patients underwent computed tomography (CT) with slices thinner than $2 \mathrm{~mm}$ and pulmonary function test (PFT). Perioperative data were collected from medical records, chest roentgenogram, CT, PFT, and endoscopic images. The exclusion criteria were: (I) right upper lobectomy with bronchoplasty or carinal resection, (II) right upper lobectomy with segmentectomy or lobectomy of other lobe, (III) middle lobar torsion, (IV) hypoplasia of RML (the volume $<100 \mathrm{~mL}$ ), and (V) past history of thoracic surgery or mediastinal radiotherapy. The study was conducted in accordance with the Declaration of Helsinki (as revised 2013). This study was approved by the Institutional Review Board of University of Tsukuba Hospital (November 11, 2019, R01-275). Informed consent was waived because of the retrospective design.

\section{Pulmonary function test (PFT) and the measurement of lung volume}

The PFT was performed in all the patients before surgery. The spirometric variables including vital capacity (VC), forced volume capacity (FVC), forced expiratory volume in one second (FEV1.0), and FEV1.0/FVC ratio were measured. The percentage of predicted VC (\%VC) and FEV1.0 (\%FEV1.0) were expressed as the percentage of the predicted value for age, gender, and height. The preoperative lung volume was evaluated using the SYNAPSE VINCENT 3D-CT software analyzer (Fujifilm, Tokyo, Japan).

\section{Operation and postoperative management}

Right upper lobectomy was performed using either videoassisted thoracic surgery or open thoracotomy under general anesthesia and one-lung ventilation. The right upper lobe (RUL) bronchus was closed at a right angle to the line of 

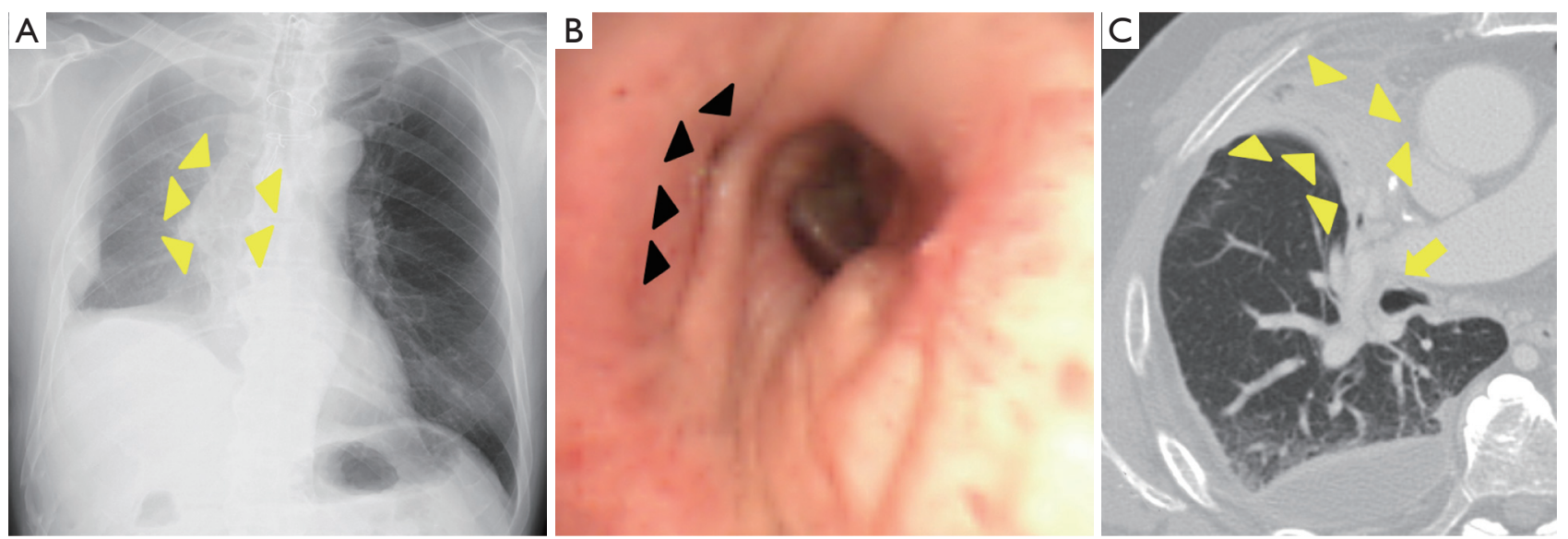

Figure 1 The findings of middle lobe bronchus kinking. (A) The X-ray photograph shows the loss aeration of the right middle lobe (arrow heads) above right hilum along the mediastinum. (B) Bronchoscopy shows a slit-like obstruction at the orifice of the middle lobe bronchus (arrowheads). (C) CT shows complete atelectasis of right middle lobe (arrowheads) and obstruction of middle lobe bronchus (arrow).

right main bronchus, parallel to the spur of second carina, using mechanical stapler. The right inferior pulmonary ligament was preserved. In the postoperative course, chest roentgenogram was routinely performed on days $1,2,4$, and 7 . Bronchoscopy was performed by a surgeon in the cases with suspected RML kinking due to abnormal chest roentgenogram findings such as atelectasis, opacification or any other findings. Bronchoscopy was also performed in the cases with diabetes or nodal dissection $2 \mathrm{a}-2$ to check for postoperative ischemic bronchitis in the early postoperative period.

\section{The definition of middle lobe bronchus kinking}

Middle lobe bronchus kinking was defined as cases that satisfied the following criteria: (I) complete atelectasis of RML (Figure 1A,B) with slit-like obstruction of the middle lobe bronchus (Figure 1C) found in the postoperative bronchoscopy, and (II) no findings suggestive of lobar torsion.

\section{The analysis of risk factors}

All the patients who satisfied the above-mentioned criteria were included in this analysis. The cohort was divided into two groups according to the presence of or absence of middle lobe bronchus kinking (kink group and no kink group, respectively) and the risk factors were analyzed. The treatment and outcome of middle lobe bronchus kinking were also extracted.

\section{The analysis of displacement of residual lung lobes}

The patients included in this analysis were only those who underwent CT with slices thinner than $2 \mathrm{~mm}$ at 6 months after right upper lobectomy during follow-up in our hospital. The distance of the middle lobe displacement was defined as A (the distance between the top of right thoracic cavity (RTC) and cranial side of RML in the preoperative CT) - B (the distance between the top of RTC and cranial side of RML in the postoperative CT) (Figure $2 A$ ). The distance of right lower lobe (RLL) displacement was defined as $\mathrm{C}$ (the distance between the top of RTC and cranial side of RLL in the preoperative CT) - D (the distance between the top of RTC and cranial side of RLL in the postoperative CT) (Figure 2B). The cranial displacement of the middle and lower lobes were compared between the kink and the no kink groups. The correlations between the displacement of residual lung lobes and the extracted factors in the risk factor analysis were also evaluated.

\section{Statistical analysis}

Each value was expressed as the mean \pm standard deviation. Chi square test or Fisher exact test were used for categorical values. Mann-Whitney test was used to compare continuous variables. Sensitivity, Specificity, optimal cut off value, and areas under the curves (AUC) were calculated using receiver operating characteristic (ROC) curves. Spearman rank correlation coefficient was used to assess the correlation of two variables. Data were analyzed using SPSS version 25.0 (IBM Corporation, NY, USA) and $\mathrm{P}$ values of $<0.05$ were 
A

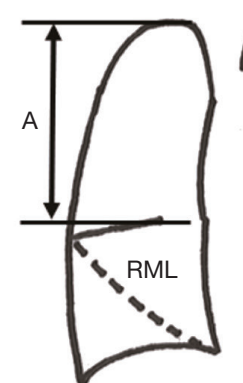

Before operation

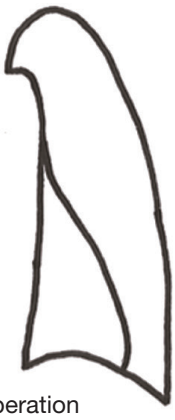

The distance of RML displacement $=A-B$

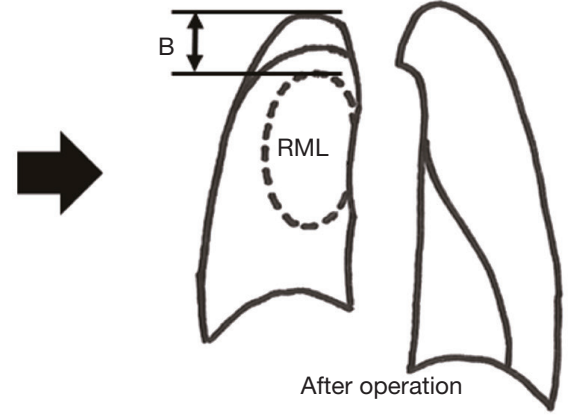

After operation
B

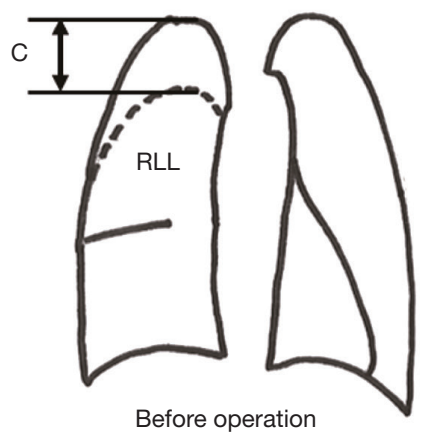

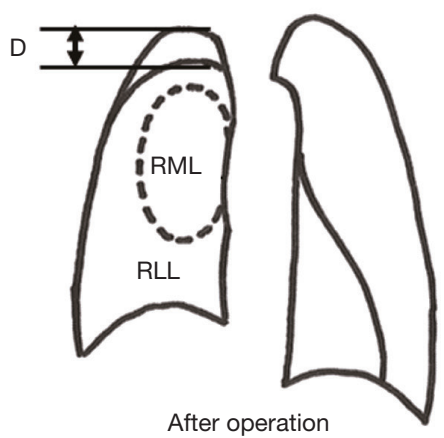

The distance of RLL displacement $=C-D$

Figure 2 The method to measure the displacement of RML and RLL. (A) The distance of middle lobe displacement was defined as A (the distance between the top of right thoracic cavity and cranial side of RML in the preoperative CT) - B (the distance between the top of right thoracic cavity and cranial side of RML in the postoperative CT). (B) The distance of RLL displacement was defined as C (the distance between the top of right thoracic cavity and cranial side of RLL in the preoperative CT) - D (the distance between the top of right thoracic cavity and cranial side of RLL in the postoperative CT). RML, Right middle lobe; RLL, right lower lobe.

considered as statistically significant.

\section{Results}

\section{Patients}

Among 209 patients who underwent right upper lobectomy, 34 patients were excluded for the following reasons: segmentectomy or lobectomy of other lobe $(n=13)$, past history of thoracic surgery or mediastinal radiotherapy (11), bronchoplasty or carinal resection (7), hypoplasia of RML (2), and middle lobar torsion (1). The remaining 175 patients met the inclusion criteria and were enrolled in this study.

\section{Risk analysis of middle lobe bronchus kinking}

\section{Patient characteristics}

Patient characteristics are shown in Table 1. Most of the patients underwent surgery for lung cancer (95\%). Bronchoscopy was performed in 71 patients $(41 \%)$ in the early postoperative period after right upper lobectomy. Middle lobe bronchus kinking was confirmed in 5 patients $(2.9 \%)$. Four out of 5 patients were symptomatic (hypoxemia, 4; breathlessness, 2; pneumonia, 1). The patient characteristics of the kink and no kink groups are shown in Table 2. There was no significant difference in age, sex, body mass index, smoking status, \% VC, FEV1.0, \%FEV1.0, surgical approach, stapler use in the horizontal fissure, and the extent of nodal dissection, between the two groups. Only FEV1.0/FVC in the kink group was significantly lower than no kink group $(\mathrm{P}=0.021)$.

\section{The risk factors analysis}

RUL volumes of the kink and no kink groups were $970.40 \pm 149.88 \mathrm{~mL}$ and $856.54 \pm 236.51 \mathrm{~mL}(\mathrm{P}=0.218)$, 
Table 1 Clinical characteristics of the patients $(n=175)$

\begin{tabular}{|c|c|}
\hline Variables & Number \\
\hline Age (years), mean \pm SD & $68.18 \pm 10.87$ \\
\hline Sex (male/female) & $99(57 \%) / 76(43 \%)$ \\
\hline BMI $\left(\mathrm{kg} / \mathrm{m}^{2}\right)$, mean $\pm \mathrm{SD}$ & $22.95 \pm 3.23$ \\
\hline Smoking (pack-years), mean \pm SD & $29.18 \pm 31.98$ \\
\hline \multicolumn{2}{|l|}{ Surgical approach } \\
\hline Open thoracotomy & $7(4 \%)$ \\
\hline Video-assisted thoracotomy & 168 (96\%) \\
\hline Stapler use in horizontal fissure & $159(91 \%)$ \\
\hline \multicolumn{2}{|l|}{ ND } \\
\hline Hilar ND & $39(22 \%)$ \\
\hline Mediastinal ND & $136(78 \%)$ \\
\hline Complication & $27(15 \%)$ \\
\hline Respiratory & $17(10 \%)$ \\
\hline Cardiac & $8(5 \%)$ \\
\hline Others & $2(1 \%)$ \\
\hline \multicolumn{2}{|l|}{ Histology } \\
\hline Primary lung cancer & $167(95 \%)$ \\
\hline Metastatic lung tumor & $6(3 \%)$ \\
\hline Benign disease & $2(1 \%)$ \\
\hline Bronchoscopy & $71(41 \%)$ \\
\hline Postoperative CT in 6 months after operation & $83(47 \%)$ \\
\hline
\end{tabular}

BMI, body mass index; ND, nodal dissection.

respectively. RML volumes of the kink and no kink groups were $322.80 \pm 87.15 \mathrm{~mL}$ and $423.64 \pm 133.00 \mathrm{~mL}(\mathrm{P}=0.066)$, respectively. On the other hand, the volume ratio of RML/RTC of the kink group was significantly lower than that of the no kink group $(0.14 \pm 0.02$ vs. $0.19 \pm 0.05$, $\mathrm{P}=0.016$ ), and that of RML/RUL of the kink group was significantly lower than that of the no kink group $(0.33 \pm 0.08$ vs. $0.52 \pm 0.18, \mathrm{P}=0.006)$, respectively, as shown Table 3. The ROC curves of RML/RTC and RML/RUL as risk factors are shown in Figure 3. The Youdan index was selected to calculate the optimal cutoff values in the ROC curve. The optimal cutoff values of RML/RTC and RML/RUL were 0.149 (AUC 0.815 , specificity 0.800, sensitivity 0.818 ), and 0.312 (AUC 0.864 , specificity 0.600 , sensitivity 0.965 ).
The treatment and outcome of middle bronchus kinking

All 5 patients with middle lobe bronchus kinking were treated with aeration to the RML using bronchoscope under fluoroscopy. Following the treatment, RML aeration was maintained only in 1 patient; in 3 patients, RML atelectasis spontaneously improved on CT 6 months later; and in 1 patient, RML atelectasis did not improve on CT even after 6 months. No complication occurred following RML aeration. Consequently, postoperative hospital stay in the kink group was significantly longer than the no kink group $(23 \pm 19.20$ vs. $12.16 \pm 23.38, \mathrm{P}=0.004)$.

The analysis of the displacement of residual lung lobes For this analysis, 83 patients who underwent CT at 6 months ( \pm 15 days) after surgery in our hospital were enrolled ( 3 in the kink group and 80 in the no kink group). A total of 92 patients were excluded because of significant differences of the timing of CT or follow-up in other hospital. Table 4 shows the comparison between the degree of cranial displacement of residual RML and RLL in both groups. The displacement of RML in the kink group was significantly more distant than in the no kink group $(\mathrm{P}=0.019)$, while that of RLL was not $(\mathrm{P}=0.127)$. Figure 4 shows the correlations between the preoperative volume ratio of RML to RTC (RML/RTC) or that of RML to RUL (RML/RUL), and the distance of displacement toward the upper part of residual RML and RLL. RML/ RUL had a moderate inversed correlation with the distance of displacement of RML (Figure $4 A, \mathrm{r}=-0.547, \mathrm{P}<0.001$ ) and RLL (Figure $4 B, \mathrm{r}=-0.470, \mathrm{P}<0.001$ ). RML/RTC had a mild inversed correlation with the distance of displacement of RML (Figure 4C, r=-0.282, $\mathrm{P}=0.010$ ) and RLL (Figure 4D, $\mathrm{r}=-0.274, \mathrm{P}=0.012)$.

\section{Discussion}

Bronchial kinking is thought to be caused by the displacement of the residual lung lobes. The incidence of bronchial kinking in the RML after right upper lobectomy is more frequent compared to those after other lobectomies because of anatomical features $(4,5)$. Prevention methods and effective treatments for bronchial kinking have not yet been established despite the many associated symptoms such as persistent cough, breathlessness, and inflammation, even atelectasis, pneumonia, and decrease of pulmonary function $(4,8,9)$. Therefore, our investigation of the risk factors and etiology of bronchial kinking is an important 
Table 2 Comparison of the characteristics with $(\mathrm{n}=5)$ or without $(\mathrm{n}=170)$ middle lobe bronchus kinking

\begin{tabular}{|c|c|c|c|}
\hline Variables & Kink group $(n=5)$ & No kink group $(n=170)$ & $P$ value \\
\hline Sex & & & 0.623 \\
\hline Male & $3(60 \%)$ & $96(56 \%)$ & \\
\hline Female & $2(40 \%)$ & $74(44 \%)$ & \\
\hline BMI $\left(\mathrm{kg} / \mathrm{m}^{2}\right)$, mean $\pm \mathrm{SD}$ & $23.74 \pm 2.40$ & $22.93 \pm 3.26$ & 0.457 \\
\hline \multicolumn{4}{|l|}{ Pulmonary function test, mean \pm SD } \\
\hline$\%$ VC (\%) & $108.34 \pm 15.12$ & $105.88 \pm 16.11$ & 0.792 \\
\hline FEV1.0 (mL) & $2,052 \pm 442.69$ & $2,314.70 \pm 614.29$ & 0.359 \\
\hline \%FEV1.0 (\%) & $85.18 \pm 6.64$ & $97.23 \pm 20.08$ & 0.069 \\
\hline Open thoracotomy & $1(20 \%)$ & $38(22 \%)$ & \\
\hline Video-assisted thoracotomy & $4(80 \%)$ & $132(78 \%)$ & \\
\hline Stapler use in horizontal fissure & $5(100 \%)$ & $154(91 \%)$ & 0.616 \\
\hline ND & & & 0.691 \\
\hline Hilar ND & $1(20 \%)$ & $38(22 \%)$ & \\
\hline Mediastinal ND & $4(80 \%)$ & $132(78 \%)$ & \\
\hline Postoperative hospital stay (day), mean \pm SD & $23.00 \pm 19.20$ & $12.16 \pm 23.38$ & 0.004 \\
\hline
\end{tabular}

Chi square test or Fisher exact test were used for categorical values. Mann-Whitney $U$ test was used to compare continuous variables. BMI, Body mass index; FEV1.0, forced expiratory volume in 1 second; FVC, functional volume capacity; ND, nodal dissection; \%FEV1.0, the percentage of predicted forced expiratory volume in 1 second; \% VC, the percentage of predicted vital capacity.

Table 3 Comparison of the volume and volume ratio of each lung lobe with $(n=5)$ or without $(n=170)$ middle lobe bronchus kinking

\begin{tabular}{|c|c|c|c|}
\hline Characteristics & Kink group $(n=5)$ & No kink group $(n=170)$ & $P$ value \\
\hline \multicolumn{4}{|c|}{ Lung volume (mL) } \\
\hline RUL & $970.40 \pm 149.88$ & $856.54 \pm 236.51$ & 0.218 \\
\hline RML & $322.80 \pm 87.15$ & $423.64 \pm 133.00$ & 0.066 \\
\hline RML/RUL & $0.33 \pm 0.08$ & $0.52 \pm 0.18$ & 0.006 \\
\hline RUL/RLL & $1.08 \pm 0.28$ & $0.92 \pm 0.45$ & 0.113 \\
\hline RML/RLL & $0.35 \pm 0.08$ & $0.45 \pm 0.19$ & 0.194 \\
\hline RUL/RTC & $0.44 \pm 0.06$ & $0.38 \pm 0.08$ & 0.052 \\
\hline
\end{tabular}

Mann-Whitney $\mathrm{U}$ test was used for statistical analysis. RLL, right lower lobe; RML, right middle lobe; RTC, right thoracic cavity; RUL, right upper lobe. 
A

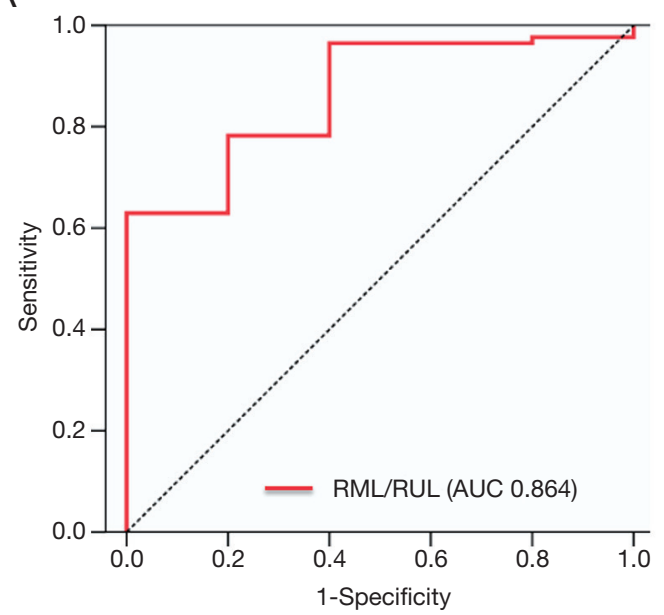

B

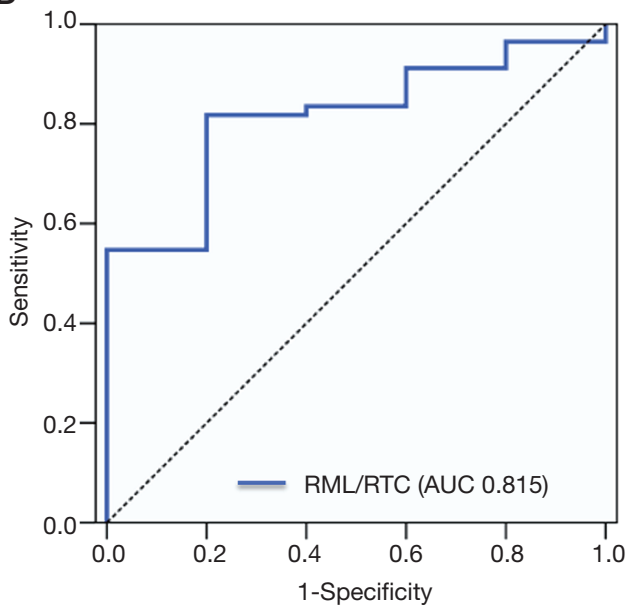

Figure 3 ROC curves of RML/RUL (red line) and RML/RTC (blue line) as risk factors for middle lobe bronchus kinking. (A) The optimal cutoff values of RML/RUL were 0.312 (AUC 0.864, specificity 0.600 , sensitivity 0.965 ). (B) The optimal cutoff values of RML/RTC were 0.149 (AUC 0.815, specificity 0.800, sensitivity 0.818). ROC, receiver operating characteristics; RTC, right thoracic cavity; RML, right middle lobe; RUL, right upper lobe; AUC, area under the curves.

Table 4 Comparison of the displacement of residual lobe toward upper side with $(\mathrm{n}=3)$ or without $(\mathrm{n}=80)$ middle lobe

\begin{tabular}{|c|c|c|c|}
\hline Variables & Kink group $(n=3)$ & No kink group $(\mathrm{n}=80)$ & $P$ value \\
\hline Displacement of RML (mm) & $120.67 \pm 13.05$ & $96.19 \pm 18.01$ & 0.019 \\
\hline
\end{tabular}

Mann-Whitney U test was used for statistical analysis. RML, Right middle lobe; RLL, right lower lobe.

step towards prevention of the negative consequences on the patients' long-term quality of life. In a study by Ueda et al., where RML displacement after right upper lobectomy was divided into two types based on whether the RML excessively migrated to the cranial side or not, the type of migration associated with the incidence of middle lobe bronchus kinking remained unclear (12). Hence, we considered two possible causes of middle lobe bronchus kinking based on the displacement by different residual lobes: (I) the kinking is caused by the cranial displacement of the RML or (II) it is caused by the RML being compressed ventrally due to the cranial displacement of the RLL. Additionally, the volume of each lung lobe was also presumed to be associated with the types and degrees of displacement following right upper lobectomy. We hypothesized that the displacement of residual lung lobe after lobectomy can depend on the volume of each lung lobe. Thus, we investigated the risk factors of middle lobe bronchus kinking and the displacement of the residual lobes after right upper lobectomy focusing on the volume of each lung lobe.

In this study, the frequency of middle lobe bronchial kinking after right upper lobectomy was approximately $2.9 \%$, which is lower than previous reports (11\% and $42 \%)$ $(8,9)$. This discrepancy might result from ambiguities over the definition of bronchial kinking used in the respective papers, such as whether middle lobe atelectasis was combined or not. In this study, middle lobe bronchus kinking was defined as the presence of a slit-like obstruction of the middle lobe bronchus and complete atelectasis of RML, because the most problematic result of middle lobe bronchus kinking is atelectasis which can lead to symptoms such as pneumonia and hypoxia. Therefore, the incidence rate of middle lobe bronchus kinking in this study was estimated to be lower than that of previous studies. Also, the low FEV1.0/FVC was the only risk factor observed among the general characteristics. One previous study showed that the presence of chronic obstructive pulmonary disease 
A

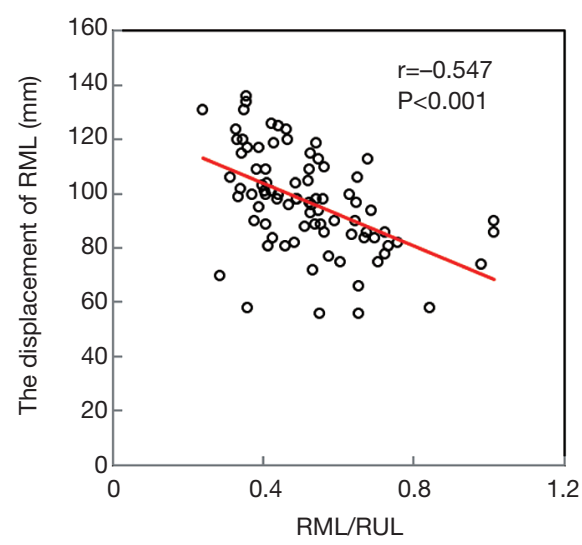

C

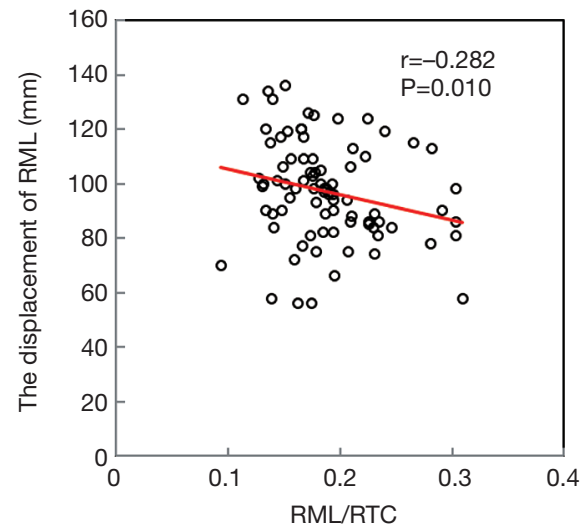

B

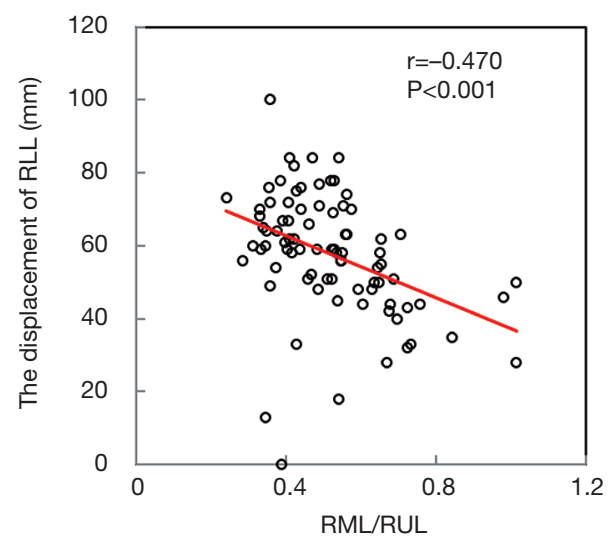

D

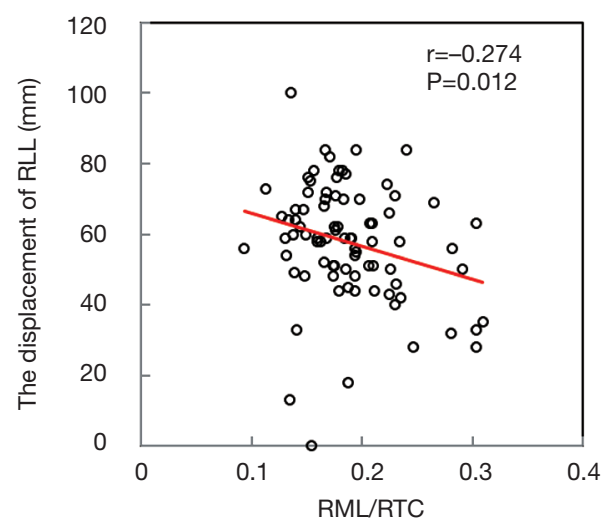

Figure 4 Correlations between the displacement toward cranial of the residual lobes and both RML/RUL and RML/RTC. (A,B) RML/ RUL had a moderate inversed correlation with the displacement toward cranial of RML $(r=-0.547, \mathrm{P}<0.001)$ and RLL ( $r=-0.470$, $\mathrm{P}<0.001)$. (C,D) RML/RTC had a mild inversed correlation with that of RML ( $r=-0.282, \mathrm{P}=0.010)$ and RLL $(\mathrm{r}=-0.274, \mathrm{P}=0.012)$. red line: approximately straight line. RML, right middle lobe; RUL, right upper lobe; RTC, right thoracic cavity; RLL, right lower lobe.

(COPD) was a risk factor of middle lobe bronchus kinking, which was in concordance with our finding of low FEV1.0/ FVC as a risk factor $(4,8)$.

The low volume ratios of RML to RTC and RML to RUL were significant risk factors for middle lobe bronchus kinking after right upper lobectomy, which suggested that a small RML, a large RTC, and a large RUL were risk factors. On the other hand, there was no significant difference in the simple volume of each lung lobe. The volume of each lung lobe is thought to be influenced by many factors such as sex, body size, coexisting lung disease, and smoking history; however, by comparing the ratios of one lung lobe to another we can, arguably, avoid any complications from these factors, which could result in the detection of more subtle risk factors. Although the routine calculation of these volume ratios might not be practical in the clinical settings, we suggest that calculating these indicators could be useful to predict the occurrence of middle lobe bronchus kinking after right upper lobectomy in the cases with relatively large RUL or relatively small RML.

The following mechanisms are thought to be involved in filling the residual space that occurs after right upper lobectomy: (I) overexpansion and displacement of residual lung lobe, (II) mediastinum shift to the affected side, and (III) elevation of the diaphragm. This displacement is considered to be the cause of middle lobe bronchus kinking following right upper lobectomy $(5,9,10,13)$. In fact, the analysis of displacement showed the cranial displacement of RML at 6 months after surgery was significantly more distant in the kink group than the no kink group, while that of RLL was not significant. The results also showed that the volume ratio of RML to RUL had a moderate inverted correlation with the displacement toward the upper part of RML and RLL. Also, the ratio of RML to RTC had a mild 
inverted correlation only with the displacement toward the upper part of RML and RLL. These findings suggested that the displacement toward the upper part of RML occurred in patients with low volume ratios of RML/ RTC and RML/RUL, in other words, in those at highrisk of middle lobe bronchus kinking. Taken together, the displacement of residual lobe, especially RML, could be related to the incidence of middle lobe bronchus kinking after right upper lobectomy. In addition, other studies have revealed that dissection of the inferior pulmonary ligament during a right upper lobectomy made the bronchial angle steeper and had a negative effect on postoperative lung function (14-17). The inferior pulmonary ligament generally stabilizes the RLL and mediastinal structure; therefore, dissection of the inferior pulmonary ligament could contribute to RLL displacement. In terms of etiology in cases of small RML, the RLL could migrate toward the vacant space to compensate the reduced RML volume. Consequently, the remaining RML might be compressed cranially by the RLL and, thereby, cause the kinking of the middle lobe bronchus.

Two methods to prevent middle lobe bronchus kinking can be envisioned: (I) fixation of RML to prevent migration to cranial side and (II) reduction of migration by somehow filling in the residual space. The former was previously reported with various method as the prevention for RML torsion (18). In fact, the degree of cranial displacement of RML was longer than that of RLL. Therefore, the fixation of RML to RLL may reduce the cranial displacement of RML and prevent middle lobe bronchus kinking. The latter might keep the residual lobes close to their original positions and prevent kinking of the middle lobe bronchus (9). In fact, patients with post pneumonectomy syndrome could improve with treatment involving filling the thoracic cavity with artifacts for lessening the degree of displacement $(19,20)$. However, this method is not reasonable in consideration of the low frequency and mild symptoms associated with middle lobe bronchus kinking, and the risk of infection with the artifacts used for filling. As a more practical method, we conjectured that pleural tenting or using a pericardial fat pad can be useful, although we did not try in this study. Pleural tenting can be used after right upper lobectomy to separate the upper space from the residual thoracic cavity as an extra pleural pocket. Consequently, the residual lung lobe could stay close to the original space (21). Although using a pericardial fat pad is a simple method to fill the residual space, a fat pad of large volume is needed to keep the residual lung lobe close to its original position. Further studies are required to assess the effectiveness of these methods.

In our hospital, all patients with middle lobe bronchus kinking were treated with aeration to the RML. As a result, RML atelectasis improved in 1 patient during the treatment, 3 patients improved spontaneously within 6 months of follow-up, and 1 patient was not improved even after 6 months follow-up. Although no complication occurred following the aeration in our experience, RML atelectasis improved only 1 of 5 patients, which suggests the limited efficacy of aeration, although more studies would be needed to confirm this. Moreover, most of the patients in our study improved spontaneously. Thus, long-term remodeling in the thoracic cavity could improve middle bronchus kinking.

There are three limitations in this study. Firstly, it was a retrospective study carried out at a single institution. Secondly, 92 patients were excluded in the correlation analysis because of differences of the timing (more than 16 days) of CT, because this time difference could have had some bearing on the degree of the displacement. Finally, in this study, we evaluated the displacement of the residual lung lobes only towards the cranial side. Future studies that can evaluate the movement of residual lung lobes with three-dimensional analysis are anticipated, which could allow us to get more detailed information about the displacement of residual lobes.

\section{Conclusions}

The risk of middle lobe bronchus kinking is associated with the volume ratio of RML to RTC and the ratio of RML to RUL. We observed that the displacement towards the upper part of the RML is associated with the incidence of middle lobe bronchus kinking. Future studies that can calculate the movement of residual lung lobes using three-dimensional analysis would be useful to fully elaborate the mechanisms of bronchus kinking.

\section{Acknowledgments}

We appreciate Thomas Mayers, Medical English Communications Center, University of Tsukuba, for revision of this manuscript.

Funding: None.

\section{Footnote}

Reporting Checklist: The authors have completed the 
STROBE reporting checklist. Available at http://dx.doi. org/10.21037/jtd-21-105

Data Sharing Statement: Available at http://dx.doi. org/10.21037/jtd-21-105

Peer Review File: Available at http://dx.doi.org/10.21037/jtd21-105

Conflicts of Interest: All authors have completed the ICMJE uniform disclosure form (available at http://dx.doi. org/10.21037/jtd-21-105). The authors have no conflicts of interest to declare.

Ethical Statement: The authors are accountable for all aspects of the work in ensuring that questions related to the accuracy or integrity of any part of the work are appropriately investigated and resolved. The study was conducted in accordance with the Declaration of Helsinki (as revised 2013). This study was approved by the institutional review board of University of Tsukuba Hospital (November 11, 2019, R01-275). Informed consent was waived because of the retrospective design.

Open Access Statement: This is an Open Access article distributed in accordance with the Creative Commons Attribution-NonCommercial-NoDerivs 4.0 International License (CC BY-NC-ND 4.0), which permits the noncommercial replication and distribution of the article with the strict proviso that no changes or edits are made and the original work is properly cited (including links to both the formal publication through the relevant DOI and the license). See: https://creativecommons.org/licenses/by-nc-nd/4.0/.

\section{References}

1. Ginsberg RJ, Rubinstein LV. Randomized trial of lobectomy versus limited resection for T1 N0 non-small cell lung cancer. Lung Cancer Study Group. Ann Thorac Surg 1995;60:615-22; discussion 622-3.

2. Arslantas MK, Kara HV, Tuncer BB, et al. Effect of the amount of intraoperative fluid administration on postoperative pulmonary complications following anatomic lung resections. J Thorac Cardiovasc Surg 2015;149:31420, 21.e1.

3. Agostini PJ, Lugg ST, Adams K, et al. Risk factors and short-term outcomes of postoperative pulmonary complications after VATS lobectomy. J Cardiothorac Surg
2018;13:28.

4. Stolz AJ, Schutzner J, Lischke R, et al. Predictors of atelectasis after pulmonary lobectomy. Surg Today 2008;38:987-92.

5. Korst RJ, Humphrey CB. Complete lobar collapse following pulmonary lobectomy. Its incidence, predisposing factors, and clinical ramifications. Chest 1997;111:1285-9.

6. Dai J, Xie D, Wang H, et al. Predictors of survival in lung torsion: A systematic review and pooled analysis. J Thorac Cardiovasc Surg 2016;152:737-745.e3.

7. Felson B. Lung torsion: radiographic findings in nine cases. Radiology 1987;162:631-8.

8. Masuda Y, Marutsuka T, Suzuki M. A risk factor for kinked middle lobar bronchus following right upper lobectomy. Asian Cardiovasc Thorac Ann 2014;22:955-9.

9. Ueda K, Tanaka T, Hayashi M, et al. Clinical ramifications of bronchial kink after upper lobectomy. Ann Thorac Surg 2012;93:259-65.

10. Gu Q, Qi S, Yue Y, et al. Structural and functional alterations of the tracheobronchial tree after left upper pulmonary lobectomy for lung cancer. Biomed Eng Online 2019;18:105.

11. Seok Y, Cho S, Lee JY, et al. The effect of postoperative change in bronchial angle on postoperative pulmonary function after upper lobectomy in lung cancer patients. Interact Cardiovasc Thorac Surg 2014;18:183-8.

12. Ueda K, Tanaka T, Hayashi M, et al. Right middle lobe transposition after upper lobectomy: influence on postoperative pulmonary function. Thorac Cardiovasc Surg 2013;61:138-43.

13. Van Leuven M, Clayman JA, Snow N. Bronchial obstruction after upper lobectomy: kinked bronchus relieved by stenting. Ann Thorac Surg 1999;68:235-7.

14. Bu L, Yang AR, Peng H, et al. Dividing inferior pulmonary ligament may change the bronchial angle. J Surg Res 2016;201:208-12.

15. Usuda K, Sagawa M, Aikawa H, et al. Do Japanese thoracic surgeons think that dissection of the pulmonary ligament is necessary after an upper lobectomy? Surg Today 2010;40:1097-9.

16. Seok Y, Yi E, Cho S, et al. Perioperative outcomes of upper lobectomy according to preservation or division of the inferior pulmonary ligament. J Thorac Dis 2015;7:2033-40.

17. Kim DH, Moon DH, Kim HR, et al. Effect of inferior pulmonary ligament division on residual lung volume and function after a right upper lobectomy. Interact Cardiovasc 
Thorac Surg 2019;28:760-6.

18. Uramoto H, Takenoyama M, Hanagiri T. Simple prophylactic fixation for lung torsion. Ann Thorac Surg 2010;90:2028-30.

19. Soll C, Hahnloser D, Frauenfelder T, et al. The postpneumonectomy syndrome: clinical presentation and treatment. Eur J Cardiothorac Surg 2009;35:319-24.

Cite this article as: Yanagihara T, Sekine Y, Sugai K, Kawamura T, Maki N, Saeki Y, Kitazawa S, Kobayashi N, Kikuchi S, Goto Y, Ichimura H, Sato Y. Risk factors of middle lobe bronchus kinking following right upper lobectomy. J Thorac Dis 2021;13(5):3010-3020. doi: 10.21037/jtd-21-105
20. Shen KR, Wain JC, Wright CD, et al. Postpneumonectomy syndrome: surgical management and long-term results. J Thorac Cardiovasc Surg 2008;135:1210-6; discussion 1216-9.

21. Venuta F, De Giacomo T, Rendina EA, et al. Thoracoscopic pleural tent. Ann Thorac Surg 1998;66:1833-4. 